\title{
Gestión de la información y comunicación en hospitales públicos: una revisión de literatura
}

Information and communication management in public hospitals: a literature review

Gestão da informação e comunicação em hospitais públicos: uma revisão da literatura

Karla Andrea Santellán Pinedo

kasantellanp@ucvvirtual.edu.pe

Universidad Cesar Vallejo - Perú

https://orcid.org/0000-0002-3917-7044

Gabriela del Pilar Palomino Alvarado

dpalominoal@ucvvirtual.edu.pe

Universidad Cesar Vallejo - Perú

http://orcid.org/0000-0002-2126-2769

\author{
Franz Jhonn Vargas Guzmán \\ fvarguz@gmail.com \\ Universidad Cesar Vallejo - Perú \\ https://orcid.org/0000-0002-1396-5229
}

\begin{abstract}
RESUMEN
La investigación tuvo como objetivo conocer los pilares fundamentales para la gestión de la información y comunicación en hospitales públicos, la cual se desarrolló mediante el método de revisión bibliográfica de diversos artículos científicos en español, inglés y portugués, tomando como criterio la selección de estudios con antigüedad no mayor a cinco años; las cuales se encuentran disponibles en la base de datos como Dialnet, SciELO, PubMed, ResearchGate y Redalyc. Proceso que consistió en realizar un análisis de coherencia y similitud de contenido, logrando obtener como resultados que los 30 articulo estudiados. Como evidencia de ello, se ha logrado identificar cuatro ejes principales, como son las herramientas de comunicación, además de las estrategias de comunicación, las características de comunicación para mantener los registros seguros, por último, y no menos importante el eje denominado competencias digitales. Logrando concluir que, la gestión de la información y comunicación presenta diversas características fundamentales para el desarrollo de los procedimientos hospitalarios, por tanto, se caracterizan por ser utilices, fáciles, seguros y confiables.
\end{abstract}

Palabras claves: Gestión, comunicación, hospitales públicos.

\begin{abstract}
The objective of the research was to know the fundamental pillars for the management of information and communication in public hospitals, which was developed through the method of bibliographic review of various scientific articles in Spanish, English and Portuguese, taking as a criterion the selection of studies with no more than five years old; which are available in the database as Dialnet, SciELO, PubMed, ResearchGate and Redalyc. Process that consisted of carrying out an analysis of coherence and similarity of content, obtaining as results that the 30 articles studied. As evidence of this, it has been possible to identify four main axes, such as communication tools, in addition to communication strategies, communication characteristics to keep records safe, last but not least the axis called digital skills. Managing to conclude that the management of information and communication presents various fundamental characteristics for the development of hospital procedures, therefore, they are characterized by being useful, easy, safe and reliable.
\end{abstract}

Keywords: Management, communication, public hospitals.

\section{RESUMO}


O objetivo da pesquisa foi conhecer os pilares fundamentais para a gestão da informação e comunicação em hospitais públicos, a qual foi desenvolvida através do método de revisão bibliográfica de diversos artigos científicos em espanhol, inglês e português, tendo como critério a seleção dos estudos. com no máximo cinco anos; que estão disponíveis na base de dados como Dialnet, SciELO, PubMed, ResearchGate e Redalyc. Processo que consistiu em realizar uma análise de coerência e similaridade de conteúdo, obtendo como resultados os 30 artigos estudados. Como evidência disso, foi possível identificar quatro eixos principais, como as ferramentas de comunicação, além das estratégias de comunicação, as características da comunicação para manter os registros seguros, por último, mas não menos importante, o eixo denominado competências digitais. Conseguindo concluir que a gestão da informação e comunicação apresenta várias características fundamentais para o desenvolvimento dos procedimentos hospitalares, portanto, caracterizam-se por serem úteis, fáceis, seguros e fiáveis.

Palavras-chave: Gestão, comunicação, hospitais públicos.

\section{INTRODUCCIÓN}

Los niveles de competitividad y los altos requerimientos de un mundo cada vez más globalizado, "exige a todas las organizaciones como en el sector salud el uso obligatorio de herramientas de gestión de información y comunicación que les permitan manejar grandes cantidades de información diarias, para de este modo ser más competentes y eficientes en su labor" (Izhar, Ma, \& Aasir, 2017, p. 3). Sin embargo, aún existen países que no ha logrado la implementación de herramientas tecnológicas, pues "el mundo digital no llega aun a todos por igual, como es el caso de los países de África, en donde, el 60\% de personas aun no tienen la posibilidad de conectarse a internet. Por el contrario, las personas de los países europeos mantienen un porcentaje reducido (4\%)" (El País, 2019, párr. 2); situación negativa que se debe a la falta de implementación o asimilación de sistemas en los hospitales públicos que permitan el eficiente manejo de la información y la comunicación de las actividades y operaciones del día a día. (Fereshteh \& Abbas, 2016).

Al mismo tiempo, Martín (2019) refiere que, hoy en día no se concibe una organización que no plantee esquemas de comunicación que vayan a utilizarse para relacionarse con su entorno, pues dicha estrategia resulta vital a la hora de buscar ser más competitivo y eficiente en el mercado y sus procesos, la implementación de tecnologías de última generación implica ahorrarle grandes cantidades de tiempo a los colaboradores permitiendo a éste, poder emplear dicho tiempo en otras actividades de interés para la empresa o institución (Bigahash, Alexander, \& Hagen, 2020). En la misma línea, Martin-Fumadó, Morlans, y Torralbad (2019) refieren que algunas de las estrategias para evitar los desajustes derivados de una adecuada comunicación interna son; la aplicación de encuestas en todas las instancias de la entidad para conocer las necesidades del colaborador, potenciar las reuniones con el fin de fomentar la comunicación horizontal, implantar programas de convivencia entre los trabajadores, desarrollar programas de mejora de habilidades, y tomar decisiones democráticas. (Charry, 2018; Quispe, Padilla, \& Telot, 2017).

Rodríguez y Del Pino (2017) explican por su parte que, la tecnología juega un papel determinante en todas las estrategias de implementación de un modelo de gestión de la información y comunicación, ya que esta es muy cambiante, por lo cual implica una constante adecuación del talento humano a los nuevos requerimientos de dichas tecnologías (Sánchez, Montenegro, \& Medina, 2019, p.98). Sin embargo, los niveles de usabilidad y amigabilidad de los sistemas son cada día mejores, por lo que hoy le resulta más sencillo a un recién llegado manejar un software actual que un software antiguo y poco amigable. (Stanford, Schwartz, \& Khan, 2020). Asimismo, Hernández, Ramírez y Laguado, (2019) señala que para lograrlo es importante tener en cuenta los requerimientos informáticos en relación a las actividades y procedimientos de la organización, asimismo mejorar los medios para la comunicación y accesibilidad a la información, mejorar los procedimientos informativos y lograr el uso 
eficiente de las herramientas. (Salas, y otros, 2020)

Además, cabe precisar que debido al manejo de gran cantidad y volumen de información de las diferentes actividades cotidianas, las organizaciones se han visto en la necesidad de adaptar a los sistemas de información y comunicación modelos de programación que permitan el almacenamiento y procesamiento de gran cantidad de información (Parra-Rodriguez, Pavón-Sicilia \& GuerreroRodriguez, 2016), es ahí, donde el Big Data se ha convertido una tendencia en todo el mundo, ya que, es una programación que facilita la gestión inteligente de la información, ofreciendo velocidad en el procesamiento de datos y rapidez en la obtención de información a partir de las diversas variables e indicadores que se deseen analizar. (Hernández-Leal, Duque-Méndezy y Moreno-Cadavid, 2017).

Presentada la situación línea arriba, a través del presente artículo de revisión se pretendió conocer los pilares fundamentales para la gestión de la información y comunicación en hospitales públicos. Por lo tanto, de aquí en adelante se analizan fundamentos teóricos que enriquecen el conocimiento del lector.

\section{REVISIÓN TEÓRICA}

Como parte de la investigación, es importante considerar las teorías relacionadas al tema, es así que abordaremos sobre el modelo de gestión de información y comunicación, el cual es definido por Arévalo (2007) citado por Muñoz, Díaz, y Gallego, (2020), como la explotación de información para el logro de objetivos institucionales, considerando desde la indagación, procesamiento, análisis y difusión de la misma. Es decir, "la gestión permite ofrecer componentes que faciliten a la organización obtener, generar y transmitir, datos con calidad, exactitud, y actualidad al menor costo posible" (Suárez y Cuz, 2015, p. 74). Los cuales sirvan para el logro de metas, que en definitiva busca conseguir información adecuada, en el momento necesario para la toma de decisiones. (Wagner, Bezaidenhout, \& Roos, 2014).

Por su parte, Rodríguez y Del Pino (2017), señalan que la información y comunicación se refieren a componentes clave para el avance de las asociaciones contemporáneas. Por lo tanto, cabe precisar que "los ángulos identificados con intensidad, mejora persistente, situación, avance, competencia y rentabilidad, dependen en gran medida de la poderosa utilización de datos jerárquicos y formas adecuadas de correspondencia institucional" (Courtney \& Steele, p. 95). Por otro lado, CanoPita (2018), señalan que la información y la comunicación, ha cambiado el método de trabajo y supervisa los activos, es un componente clave para hacer que el trabajo sea cada vez más beneficioso, acelera los intercambios, apoya la colaboración, supervisa las acciones, realiza un examen relacionado con el dinero y artículos avanzados en el mercado. (Izzatty, Nurul, Hazana, \& Shamsuddi, 2015).

Para Yu (2020) resulta indispensable identificar nuevas modalidades de organización que dejen gestionar la información en las instituciones, pues el objetivo de estas instituciones es crear conocimiento a base de la información y transformarlas en una ventaja razonable y calculada en cuanto a la productividad. Baharun, Hefniy, Fauzi, Faridy, \& Fatmasari (2018) señalan que el sistema de información debe tener como objetivo fomentar en las organizaciones un ambiente de reciprocidad, caracterización y generación de nuevos conocimientos, datos e información interna, por lo tanto, debe organizarse de manera efectiva, para que puedan ser utilizados en el instante exacto por quienes lo necesitan, de existir la posibilidad de organizar la información conforma a los conocimiento, se logrará excelentes resultados en poco tiempo (Hernández, Ramírez y Laguado, 2019).

Por otro lado, autores como Bouchard (2017) señala que la efectividad de la gestión de 
información y comunicación dependerá de cómo se realiza el ingreso de información, proceso a través del cual el método de información adquiere datos para procesar la información, y estos pueden ser manuales y automáticas (Adombilla, 2018). Asimismo, está la acumulación de información, una de las actividades más significativas, que a través del sistema los datos pueden ser guardados en estructuras denominadas archivos (Lawan, 2019, p. 72). También se considera el procesamiento de información, este se caracteriza por transformar datos útiles para la toma de decisiones, ello genera una mejor proyección financiera. (Hanum, Gogilavani, \& Mohamed, 2020). Finalmente, la salida de información es la capacidad de un procedimiento para divulgar información ingresada y procesada a los usuarios que lo requieran en una situación dada. (Muñoz, Díaz, y Gallego, 2020).

De igual manera, Baharun, Hefniy, Fauzi, Faridy, \& Fatmasari (2018) consideran que existen técnicas de indagación y restauración de datos, tecnologías que pueden ser encontrada en base de datos y datas internos que permiten la integración de información, herramientas de filtrado y personalización, la cual permite que la información conseguida en los procedimiento de búsqueda y análisis de fuentes alcance a los agentes de manera sistemática, determinada de acuerdo a los requerimientos y de manera interactiva (León, 2018). Además, He (2019) afirma que los instrumentos de análisis de información muestran la asociación e influencia entre los datos que son relevantes para la toma de una decisión de una entidad, pues proporcionan información útil para realizar un análisis interno y externo y para la obtención de ventajas competitivas. (Correa y Díaz, 2018).

Por lo tanto, para que una organización pueda ejercer una eficiente gestión de su información y comunicación, Papic (2019) explica que es necesario la implementación de mecanismos, así como de herramientas de comunicación (Yang, Saladrigas, \& Torres 2016). Del mismo modo, Papic (2019) toma en cuenta la dimensión herramientas de la comunicación para la cual debe priorizar la utilización del manual de bienvenida: Es el primer contacto del nuevo colaborador con la organización y sirve para que la inserción del mismo sea más rápida, en ella se detalla aspectos como la historia de la organización, el organigrama, políticas, normas, etc. Boletín interno: Son informaciones periódicas enviadas a los colaboradores para dar a conocer información de interés, anunciar actividades, resultados, etc. (Arévalo y Valdez, 2018). Así mismo, Red social interna: la más usada hoy en día es el WhatsApp, pero también pueden usarse otras como el MSN de Facebook, WeChat, etc. mediante esta herramienta se mantiene informado a todos los colaboradores de manera instantánea. (Papic, 2019, pp. 73-75).

De la misma forma Atarama-Rojas y Vega-Foelsche (2020) señala que el correo electrónico; es una herramienta indispensable y mediante el cual se puede transferir información de manera más formal y segura entre todos los individuos de una organización. Por su parte Chaín, Muñoz, y Más, (2008) afirman que la intranet, permite que los colaboradores de una organización puedan trabajar de manera sencilla y efectiva, colaborando en proyectos, compartiendo información, realizar videoconferencias, etc. (p.45).

En esta línea, Medina (2019) explica que la gestión de información y comunicación debe tener en cuenta las diferentes estrategias para la comunicación ascendente, descendente, horizontal y diagonal. Asimismo, la gestión de la información y comunicación también debe tener en cuenta las características que deberá de poseer la información que se transmita dentro de la organización, es por ello que se toma los principios para la información planteados por el Ministerio de Tecnologías de la Información y Comunicaciones de Colombia (2018) siendo esta las características de la información y comunicación que se encuentran relacionadas con la oportunidad: pues la información de llegar en el momento justo cuando se necesita y que permite cumplir con los tiempos planteados (Jones, Motta, y Alderete, 2016). La confiabilidad: característica de la información que hace referencia a la ausencia de errores y de desarrollar una función específica, en un contexto dado durante un tiempo establecido. La 
completitud: característica de la información de ser siempre completa a fin de trasmitir el mensaje tal cual se desea. La pertinencia: naturaleza de los datos cuando se asocia con una circunstancia general. Hay importancia si una propuesta específica se identifica con el tema del que se habla. Inesperadamente, una proposición es insignificante en el caso de que no tenga conexión con la configuración general. La utilidad: cualidad de la información de servir o de ser aprovechada para una acción específica (MinTIC - Colombia, 2018).

\section{MATERIALES Y MÉTODOS}

\section{El diseño y el sitio del estudio}

El diseño empleado para la presente investigación fue bibliográfica o documental, ya que se realizó una revisión de material bibliográfico existente con respecto al tema a estudiar.

\section{Criterios de elegibilidad}

Tipos de participantes: investigaciones que se centraron en la gestión de la información y comunicación en hospitales públicos. Tipos de estudios: se ha considerado investigación que permitan el análisis del uso y manejo de la información y comunicación en el sector salud. Tipos de resultados: los resultados que muestren la realidad sobre la gestión de la información y comunicación en hospitales. Búsqueda de estudios: El presente estudio está elaborado a partir de estudios primarios y secundarios que poseen una antigüedad menor a 5 años encontrados en la red, para ello se ejecutó una investigación manual en las bases de datos Dialnet, SciELO, PubMed, ResearchGate y Redalyc, en el mes de diciembre 2020. Selección de estudios: El proceso de selección de los estudios fue realizado mediante un análisis de coherencia y similitud de contenido con el tema emprendido y demás intereses de la investigación, es así que como primer punto se realizó un sondeo general de artículos relacionados con la variable, posteriormente se seleccionó los que estaban enmarcados en los lineamientos de la salud y finalmente se filtró los que coincidían en similares objetivos del presente estudio. (Herbas y Rocha, 2018)

\section{Resultados de la búsqueda}

Las estrategias de búsqueda empleada en el presente artículo dieron 605 referencias, no obstante, durante el proceso de selección se eliminaron 435 referencias, pues no cumplían con los criterios de inclusión tras revisar el título, resumen y conclusiones. Siendo solo 69 referencias las que fueron leídas para confirmar la elegibilidad, es así que una vez finalizado el proceso de selección se tomaron treinta artículos científicos, pues de 69 referencias analizadas se excluyeron 36 que no cumplían con los criterios de inclusión, siendo los resultados basados en los 33 artículos restantes que se presentan de forma sistemática y descriptiva, pues la naturaleza de los estudios no permite ningún otro tipo de análisis.

\section{ANALISIS DE DATOS}

Resultados

Tras analizar cada uno de los 30 artículos seleccionados se logró identificar que estos se dividen en cuatro ejes principales, las cuales se clasifican en herramientas de comunicación, estrategias, 
características de comunicación y competencias digitales.

Inicialmente las herramientas de comunicación, se basan específicamente en todos aquellos medios empleados para la gestión de la comunicación e información en hospitales públicos, es así que Barquero, Rodríguez y González (2018) en su artículo logro determinar que los hospitales recurren a diferentes fuentes informáticas como blogs (35\%), redes sociales (86\%) y las app's (80\%) para teléfonos inteligentes, lo que se significa que las TICs son fundamentales para el crecimiento de los hospitales, ya que por medio de estas facilitan al paciente gestionar sus datos médicos hasta cierto punto. Por su parte, Mendoza y Plasencia (2018) identificaron que el 70.4\% de los docentes utiliza TIC en sus clases, el $44.4 \%$ emplea herramienta de audio, imagen $(69.1 \%)$ y video $(64.2 \%)$. El PowerPoint es el material didáctico TIC más utilizado (93.8\%), además utilizan biblioteca digital (35.8\%), YouTube (39.5\%), red social (43.7\%). El 49.4\% utiliza aula virtual. Los docentes refieren una falta de capacitación en TIC (38.3\%) y solicitan capacitación en diseño de aulas virtuales (50.6\%).

Así también, Torres, Gallo, Hallo, Jaraiseh, Muriel y Fernández (2017) junto a Herrera (2019), evidenciaron que las instituciones donde desarrollaron su estudio no manejan herramientas y plataformas tecnológicas adecuadas en el sector salud, pues los sistemas que emplean tienen fallas al ser utilizadas, lo que retrasa el desarrollo de las actividades diarias, además de dificultar la toma de decisiones. Por su parte, Muriel, García, López y Sánchez (2019) señala que más del 55 \% de los profesionales nunca utilizan ordenadores o tecnologías de la información y la comunicación para la realización remota de evaluación diagnóstica o prescripción de medidas terapéuticas. A su vez Álvarez, Luna, Torres, Higuita y Rivera (2018) manifiestan que se emplearon como herramienta la tele salud, para la recuperación de la salud, detección precoz, prevención de riesgos y promoción de estilos de vida saludable, al reducir los costos de desplazamiento a los centros de salud. Para Andrade, Urgiles y Estrella (2019) las herramientas tecnológicas Risk y Excel han permitió la construcción de modelos estocásticos que facilitaron a los profesionales de salud predecir, monitorear y dar soporte en la toma de decisiones en el tratamiento y seguimiento de indicadores. Agámes, Aldana, Barreto, Santana y Caballero (2019) determinó que las herramientas más empleadas en el sector salud son web 2.0 conocidas como blogs y podcast, que tiene el potencial de complementar, mejorar y adicionar nuevas dimensiones a la colaboración de las diversas páginas web de medicina, además de contribuir al desarrollo de profesionales y servicios.

En cuanto a las estrategias de comunicación, al ser considerada una herramienta de planificación sistemática, integral y coherente de los objetivos, instrumentos, acticas, acciones y plazos de tiempo que la institución hospitalaria debe utilizar para transmitir su mensaje e imagen. Es que las instituciones hospitalarias apuestan por diseñar nuevas ideas y acciones que mejoren la gestión de comunicación e información, tal cual lo evidencia Muñoz, Díaz, Gallego (2020) quienes determinaron 2 tipos de normas, el 55\% que regulen el ciberespacio en sí mismo y el $46 \%$ que se realizan con el uso del mismo y a las que parece aplicable el derecho convencional. En este último caso nos estamos refiriendo a la aplicación del derecho ya existente a los actos realizados a través de las TIC, como es el caso de los actos médicos.

Asimismo, Adombilla (2018) señala que las estrategias de comunicación empleada le dieron como resultados que el 1,3\% de la ciudadanía ha recibido recomendaciones sobre aplicaciones móviles de salud por algún profesional sanitario. Del mismo modo, Sotillos, Buiza, Herrera, Santana (2018) obtuvo que el $73,8 \%$ estarían dispuestos a ello, así también el $68.18 \%$ tenían correos electrónicos oficiales y copiadores, mientras que el 63,64\% tenía escáneres en sus oficinas. También, el 54.55\% tenían Impresoras LaserJet y Fax en sus oficinas. Por su parte Martínez (2018) determinó que la necesidad de aumentar los niveles de escolaridad, así como implementar cursos de capacitación para la adquisición de habilidades digitales para personas en situación desfavorable como bajos niveles de 
escolaridad, adultos mayores y mujeres.

Además, Pissaia, Kunz, Moreschi, \& Rempel (2017) señalan que sus estrategias están relacionadas con la organización y planificación de sus actividades, así como con una gestión eficaz del personal basada en los principios de la atención integral que se brinda al cliente, del mismo modo Castillo, Berbey, Torre, \& López (2017) determinó que el 56\% de los proyectos de tecnología se concentran en la ciudad capital y solo el $16 \%$ en la provincia de Chiriquí. El $64 \%$ de estos proyectos están enfocados al desarrollo de sistemas de información, principalmente enfocados al registro electrónico de pacientes. Y el $60 \%$ se refiere a proyectos relacionados con la atención primaria de salud. El MINSA y CSS ambos con un $20 \%$ de participación en proyecto TIC, además se nota la dispersión de proyectos para hospitales, donde cada uno está desarrollando programas según sus necesidades o prioridades. Finalmente, García, Guzmán y Pons (2019) platearon estrategias ligadas al avance tecnológico como la gamificacion, historias digitales, portafolios y el uso de conversational fremework, un conjunto de estrategias que descubren nuevas rutas y ritmos para la formación en el campo de la enfermería que evidencia su permanente interés por actualizarse y responder a los requerimientos que marca el desarrollo de la sociedad y la cultura digital.

Por otro lado, los artículos que enfatizaron principalmente en las características de la comunicación fueron de Ferreira, De Lima, Vandresen, Gomes, Gomes, \& Lumini, (2020) quienes demostraron la utilidad y facilidad de manejo de las TIC entre los directores de enfermería de los hospitales. Las TIC proporcionan soporte para la elección de dispositivos tecnológicos en la gestión, así como su idoneidad según el perfil de la enfermera gerente. Las TIC contribuyen a optimizar el trabajo del enfermero y ayudar a mantener registros seguros sobre la atención brindada. Asimismo, Cano-Pita (2018) determino que antes de añadir la tecnología, hay que conocer bien la organización, pues se ha descubierto que el $90 \%$ de las veces, el fracaso no es por el software ni por los sistemas, sino por el escaso conocimiento de la gente sobre su propia empresa o sus procesos empresariales.

En secuencia, Medina (2019) afirma que un 89\% de los instrumentos tecnológicos son confiables para solventar las demandas de la realidad global, que también se manifiesta en los entornos locales, en términos de convocar a la participación y protagonismo para resolver las necesidades colectivas. Del mismo modo Martín (2019) señala que la tecnología móvil puede conducir a mejoras en el flujo de trabajo, fortalecer la calidad y eficiencia de la comunicación y mejorar la accesibilidad y las relaciones entre equipos. Moreno \& Herrera (2019) señala que la gestión de información se incrementó significativamente a1.60 (32\%), el nivel de satisfacción del usuario generador de la información aumentóen0.74 (14.80 \%) y los costos para generar la información se redujeron a un total de S/4.59 (99.96\%), gracias nuevos sistemas de Información (HIS), cuya duración del uso fue alta y los usuarios de estos dos centros tenían mayor experiencia en comparación con los usuarios de otras regiones del país. (Sheykhotayefeh, Safdari, Ghazisaeedi \& Mohammadzadeh, 2017).

En este sentido, Oliver- Mora y Rueda (2017) señalan que las TIC en el sistema sanitario contribuye a mejorar diferentes aspectos como la relación de confianza entre pacientes y profesionales, formación de los pacientes, constitución de redes, autonomía y capacidad, atención sanitaria personalizadas y colaboración entre los profesionales. Así también Culebro - Trujillo, Contreras, Montiel y Barragán (2016) determinaron que las fuentes de información disponibles para el uso son del $93 \%$, el $58 \%$ asegura que la velocidad de la conexión es aceptable, el $71 \%$ considera que las condiciones del equipo de cómputo son adecuadas, y el $95 \%$ afirma que los sistemas de comunicación e información mejoran la calidad de atención. Robledo (2019) plantea un modelo de atención médica intercultural, a través del uso de tecnologías de información en eslabones críticos del proceso de atención médica, donde el empleo de las TIC han mejorar los estándares de calidad en el rubro de barreras de la comunicación por grupos culturales distintos. Antúnez, Veytia, Granma (2020) afirma 
que la accesibilidad a las TIC ha incrementado en los últimos años, así como la importancia de las competencias para dar respuesta a las demandas de la sociedad actual.

Otro de los ejes centrales que permitieron evaluar la gestión de comunicación e información son las competencias digitales, pues ofrecen la posibilidad de agilizar los procesos, obtener información veraz, oportuna y confiable, lo que genera una ventaja competitiva a largo plazo, dentro de estas destacan las investigaciones realizadas por Rubio, Burrola y Ruiz (2018) quienes señalan que en el entorno laboral es importante que las instituciones cuenten con competencias del conocimiento y uso de este tipo de herramientas en su quehacer profesional con el propósito de convertirse en una ventaja sostenida dentro de un proceso de gestión, es así que entre sus competencias tomaron como base el modelo Nonaka y Takehuchi por ser el más utilizado y aceptado.

Por su parte González, Tovilla, Juárez y López (2017), señalan que el uso de las tecnologías de información y comunicación contribuye al aumento del rendimiento e incremento de conocimientos, así también Yánez, Ortiz y Espinosa (2017) como también Iñiguez-Jiménez, Cruz-Pierard y VacaCórdova (2020), determinaron que el 53\% de alumnos sostuvieron que el uso de las TICs se encontró ampliamente difundido ya que esto se percibe en la realización de trabajos escolares $(68,87 \%)$, dejando en segundo plano las redes sociales $(67,4 \%)$ y los equipos tecnológicos son manejados en un $43.33 \%$. Preciado, Hincapie y Pabón (2019) señalan que el diseño de un programa orientado a establecer mecanismos de medición en este campo desde lo académico y profesional, contribuye a mejorar el desempeño y la calidad de servicio.

En este orden de ideas, Díaz, Gorgoso, Sánchez y Riveron (2019) destacan que las tecnologías adecuadamente utilizadas facilitan la labor del trabajador y contribuyen al desarrollo cognitivo, procedimental y actitudinal del aprendizaje. Rivera, Herrera, Naranjo y Narváez (2019) señalan que para el adecuado desarrollo de un plan de gestión de riesgos deben ser implementadas políticas sobre la seguridad de la información, capacitando al personal y realizando el seguimiento y control a las mismas. Antúnez, Veytia (2020) afirman que la accesibilidad a las TIC genera competencias investigativas, permitiendo responder a la demanda de la sociedad actual; no obstante, este recurso aún debe mejorar pues se encuentra en etapa de desarrollo.

\section{DISCUSIÓN}

La gestión de la información y comunicación se fundamenta en cuatro ejes principales que son las herramientas de comunicación, estrategias, características de comunicación y competencias digitales, y que para lograr su implementación es necesario contar con instrumentos de gestión que permitan un adecuada evaluación, por lo tanto es importante un escenario propicio, centrado en el establecimiento de políticas y normas, de tal forma alcanzar la conexión e interrelación entre las personas mediante un eficiente sistema de comunicación e información.

\section{Herramientas de comunicación}

Las herramientas de comunicación e información, son los medios y mecanismo que facilitan la emisión de un mensaje a los diferentes públicos, ya sea de la institución hacia los trabajadores como también de pacientes, o viceversa; es así que autores como Barquero, Rodríguez y González (2018) sumado a Mendoza y Plasencia (2018) identifican la importancia de utilizar los medios digitales para tener una mejor comunicación y la información sea emitida como mayor velocidad y rapidez; información que en gran medida facilita el procesamiento de datos de los pacientes y enfermeros 
respecto a las actividades y procedimientos del día a día. Al mismo tiempo, Torres, Gallo, Hallo, Jaraiseh, Muriel y Fernández (2017) junto a Herrera (2019) resaltan la necesidad de implementar herramientas y plataformas tecnológicas en el sector salud, pues debido a gran cantidad de información que procesan los hospitales a diario, existe la necesidad que el sistema tenga una alta capacidad de respuesta y confiabilidad de la información emitida, pues los sistemas que emplean tienen fallas al ser utilizadas, lo que retrasa el desarrollo de las actividades diarias, además de dificultar la toma de decisiones.

Entretanto, Muriel, García, López y Sánchez (2019) señalan que más del 55 \% de los profesionales nunca utilizan ordenadores o tecnologías de la información y la comunicación para la realización remota de evaluación diagnóstica o prescripción de medidas terapéuticas. A su vez Álvarez, Luna, Torres, Higuita y Rivera (2018) manifiestan que se emplearon como herramienta la tele salud, para la recuperación de la salud, detección precoz, prevención de riesgos y promoción de estilos de vida saludable, al reducir los costos de desplazamiento a los centros de salud. Para Andrade, Urgiles y Estrella (2019) las herramientas tecnológicas Risk y Excel han permitió la construcción de modelos estocásticos que facilitaron a los profesionales de salud predecir, monitorear y dar soporte en la toma de decisiones en el tratamiento y seguimiento de indicadores. Agámes, Aldana, Barreto, Santana y Caballero (2019) determinó que las herramientas más empleadas en el sector salud son web 2.0 conocidas como blogs y podcast, que tiene el potencial de complementar, mejorar y adicionar nuevas dimensiones a la colaboración de las diversas páginas web de medicina, además de contribuir al desarrollo de profesionales y servicios.

\section{Estrategias de comunicación}

Las estrategias de información y comunicación, son elementos que permiten llegar a cada usuario de manera objetiva, es decir las estrategias de comunicación al ser consideradas una herramienta de planificación sistemática, integral y coherente de los objetivos, instrumentos, acticas, acciones y plazos de tiempo que la institución hospitalaria debe utilizar para transmitir su mensaje e imagen. Es que las instituciones hospitalarias apuestan por diseñar nuevas ideas y acciones que mejoren la gestión de comunicación e información, tal como lo sustentan Muñoz, Díaz, Gallego (2020) y Adombilla (2018) quienes ponen de manifiesto que las estrategias de comunicación se diseñan con el propósito de gestionar adecuadamente la información procesada en los sistemas, permitiendo que todo usuario que haga uso de los sistemas informáticos, pueda accedes a ella con total facilidad, además que toda información que pudiera procesarse en los sistemas sea oportunamente actualizada contribuyendo a las decisiones del usuario. Al mismo tiempo, Martínez (2018) explica que los usuarios deben recibir capacitaciones o inducción sobre el manejo de los sistemas de información de tal forma se fortalezca las habilidades tecnológicas, lo cual permitirá que toda información o datos sean procesados adecuadamente. Procedimientos y actividades de capacitación que deben ser planificadas por la institución para su adecuada aplicación, gestión que, además, busca el desarrollo adecuado de los procesos de atención a los pacientes de los hospitales.

Del mismo modo Sotillos, Buiza, Herrera, Santana (2018) sostiene que el 73,8\% estarían dispuestos a ello, así también el $68.18 \%$ tenían correos electrónicos oficiales y copiadores, mientras que el 63,64\% tenía escáneres en sus oficinas. También el $54.55 \%$ tenían Impresoras LaserJet y Fax en sus oficinas. Además, Pissaia, Kunz, Moreschi, \& Rempel (2017) señalan que sus estrategias están relacionadas con la organización y planificación de sus actividades, así como con una gestión eficaz del personal basada en los principios de la atención integral que se brinda al cliente, del mismo modo Castillo, Berbey, Torre, \& López (2017) determinó que el 56\% de los proyectos de tecnología se 
concentran en la ciudad capital y solo el $16 \%$ en la provincia de Chiriquí. El $64 \%$ de estos proyectos están enfocados al desarrollo de sistemas de información, principalmente enfocados al registro electrónico de pacientes. Y el $60 \%$ se refiere a proyectos relacionados con la atención primaria de salud. El MINSA y CSS ambos con un $20 \%$ de participación en proyecto TIC, además se nota la dispersión de proyectos para hospitales, donde cada uno está desarrollando programas según sus necesidades o prioridades.

Finalmente, García, Guzmán y Pons (2019) platearon estrategias ligadas al avance tecnológico como la gamificación, historias digitales, portafolios y el uso de conversational fremework, un conjunto de estrategias que descubren nuevas rutas y ritmos para la formación en el campo de la enfermería que evidencia su permanente interés por actualizarse y responder a los requerimientos que marca el desarrollo de la sociedad y la cultura digital.

\section{Características de la comunicación}

Por otro lado, diversos estudios como el de Ferreira, De Lima, Vandresen, Gomes, Gomes, \& Lumini, (2020), Cano-Pita (2018) y Medina (2019) ponen en evidencia que las tecnologías de información y comunicación deben cumplir con ciertas características esenciales para su eficiente funcionamiento, así como para poder cubrir la demanda de los requerimientos informáticos de la institución, por lo tanto, requieren de velocidad para procesar datos, debe ser útil para los usuarios, debe ser de fácil manejo y debe ofrecer confiabilidad en la información que analiza.

Del mismo modo, Martín (2019) señala que la tecnología móvil puede conducir a mejoras en el flujo de trabajo, fortalecer la calidad y eficiencia de la comunicación y mejorar la accesibilidad y las relaciones entre equipos. Moreno \& Herrera (2019) señala que la gestión de información se incrementó significativamente a1.60 (32\%), el nivel de satisfacción del usuario generador de la información aumentóen0.74 (14.80\%) y los costos para generar la información se redujeron a un total de S/4.59 $(99.96 \%)$, gracias nuevos sistemas de Información (HIS), cuya duración del uso fue alta y los usuarios de estos dos centros tenían mayor experiencia en comparación con los usuarios de otras regiones del país. (Sheykhotayefeh, Safdari, Ghazisaeedi \& Mohammadzadeh, 2017).

Por su vez, Oliver-Mora y Rueda (2017) señalan que las TIC en el sistema sanitario contribuye a mejorar diferentes aspectos como la relación de confianza entre pacientes y profesionales, formación de los pacientes, constitución de redes, autonomía y capacidad, atención sanitaria personalizada y colaboración entre los profesionales. Así también Culebro - Trujillo, Contreras, Montiel y Barragán (2016) determinaron que las fuentes de información disponibles para el uso son del $93 \%$, el $58 \%$ asegura que la velocidad de la conexión es aceptable, el $71 \%$ considera que las condiciones del equipo de cómputo son adecuadas, y el $95 \%$ afirma que los sistemas de comunicación e información mejoran la calidad de atención.

Seguidamente, Robledo (2019) plantea un modelo de atención médica intercultural, a través del uso de tecnologías de información en eslabones críticos del proceso de atención médica, donde el empleo de las TIC han mejorar los estándares de calidad en el rubro de barreras de la comunicación por grupos culturales distintos. Por fin, Antúnez, Veytia, Granma (2020) afirma que la accesibilidad a las TIC ha incrementado en los últimos años, así como la importancia de las competencias para dar respuesta a las demandas de la sociedad actual.

\section{Competencias digitales}


Los estudios realizados por González, Tovilla, Juárez y López (2017), sostienen que para una adecuada gestión de comunicación e información es necesario que las instituciones cuenten con profesionales competentes, con conocimientos, habilidades y capacidades, que le permitan convertirse en una ventaja sostenida dentro del proceso de gestión.

Del mismo modo Rubio, Burrola y Ruiz (2018) señalan que en el entorno laboral es importante que las instituciones cuenten con competencias del conocimiento y uso de este tipo de herramientas en su quehacer profesional con el propósito de convertirse en una ventaja sostenida dentro de un proceso de gestión, es así que entre sus competencias tomaron como base el modelo Nonaka y Takehuchi por ser el más utilizado y aceptado. Por su parte Yánez, Ortiz y Espinosa (2017) como también IñiguezJiménez, Cruz-Pierard y Vaca-Córdova (2020), determinaron que el 53\% de alumnos sostuvieron que el uso de las TICs se encontró ampliamente difundido ya que esto se percibe en la realización de trabajos escolares $(68,87 \%)$, dejando en segundo plano las redes sociales $(67,4 \%)$ y los equipos tecnológicos son manejados en un $43.33 \%$.

En seguida, Preciado, Hincapie y Pabón (2019) señalan que el diseño de un programa orientado a establecer mecanismos de medición en este campo desde lo académico y profesional, contribuye a mejorar el desempeño y la calidad de servicio. Así, el estudio de Díaz, Gorgoso, Sánchez y Riveron (2019) destacan que las tecnologías adecuadamente utilizadas facilitan la labor del trabajador y contribuyen al desarrollo cognitivo, procedimental y actitudinal del aprendizaje. Rivera, Herrera, Naranjo y Narváez (2019) señalan que para el adecuado desarrollo de un plan de gestión de riesgos deben ser implementadas políticas sobre la seguridad de la información, capacitando al personal y realizando el seguimiento y control a las mismas. Antúnez, Veytia (2020) afirman que la accesibilidad a las TIC genera competencias investigativas, permitiendo responder a la demanda de la sociedad actual, no obstante, este recurso aún debe mejorar pues se encuentra en etapa de desarrollo.

\section{CONCLUSIONES}

Se determina que las principales herramientas de información y comunicación utilizadas por el personal del sector salud, son los blogs, las redes sociales, las aplicaciones, y sistemas computarizados que se encuentran instaladas en la institución, herramientas que son fundamentales para el crecimiento personal y organizacional del personal de salud.

Al mismo tiempo, se determina que las estrategias de comunicación e información están centradas en mejorar los canales de comunicación, partiendo del entrenamiento constante del personal para mejorar sus capacidades y habilidades de manejo de las TICs, estrategias que además deben ser planificadas para su efectiva aplicación y desarrollo en un plazo determinado.

Por otro lado, se determina que la gestión de la información y comunicación presenta diversas características fundamentales para el desarrollo de los procedimientos hospitalarios, por tanto, se caracterizan por ser utilices, fáciles, seguros y confiables.

Por último, las competencias digitales, ofrecen la posibilidad de agilizar los procesos, obtener información veraz, oportuna y confiable, lo que genera una ventaja competitiva a largo plazo, mediante el diseño de un programa orientado a establecer mecanismos de medición en este campo desde lo académico y profesional. Este estudio puede dar origen a nuevas investigaciones que aborden otros recortes teóricos, por ejemplo, considerando regiones específicas o incorporando nuevas bases de datos como Scopus y Web of Science. 


\section{REFERENCIAS BIBLIOGRÁFICAS}

Adombilla, B. (2018). Assessing the Impact on the Job of Secretaries on the Use of Information Communication Technology in Public Institutions: The Case of Bolgatanga Polytechnic. Journal of Culture, Society and Development, 44(17), 1-7. doi:https://www.iiste.org/Journals/index.php/JCSD/article/view/45040/46482

Agámez, S., Aldana, M., Barreto, V., Santana, A., \& Caballero, C. (2019). Aplicación de nuevas tecnologías de la información en la enseñanza de la medicina. Salud Uninorte, 25(1), 150-171.

Álvarez, V., Luna, I., Torres, E., Higuita, A., \& Rivera, P. (2018). Tecnologías de Información y Comunicación (TIC) aplicadas en cuidados paliativos: revisión de tema. Psicooncología, 15(2), 345-360. doi:http://dx.doi.org/10.5209/PSIC.61440

Andrade, M., Urgiles, P., \& Estrella, M. (2020). Tecnologías de la información y comunicación en el desarrollo de modelos. Revista de medicina, 80(1), 31-38.

Antúnez, A., \& Veytia, M. (2020). Desarrollo de competencias investigativas y uso de herramientas tecnológicas en la gestión de información. Revista Conrado, 16(72), 96-102.

Arévalo, L. M., \& Valdez, J. C. (2018). Comunicación Internay Productividad Laboral En Las Empresas De Tarapoto. Cientifi-k, 7(1), 55-60. doi:https://doi.org/10.18050/cientifi-k.v7i1.2126

Atarama-Rojas, T., y Vega-Foelsche, D. (2020). Comunicación corporativa y branded content en Facebook: un estudio de las cuentas oficiales de las universidades peruanas. Revista de Comunicación, 191, 37-53. doi:https://doi.org/10.26441/RC19.1-2020-A3

Baharun, H., Hefniy, Fauzi, A., Faridy, \& Fatmasari, R. (2018). National assessment management based on information and communication technology and its effect on emotional intelligence learners. 1st International Conference on Advance and Scientific Innovation, 1175, 1-4. doi:10.1088/1742-6596/1175/1/012225

Barquero, M., Rodríguez, J., y Gonzálvez, J. E. (2018). Tecnologías de la Comunicación y posverdad: implicaciones para la gestión de la Comunicación Hospitalaria. Revista de Comunicación y Salud, 8(1), 85-97. doi:https://doi.org/10.35669/revistadecomunicacionysalud.2018.8(1).85-97

Bigahash, L., Alexander, K., \& Hagen, C. (2020). A Model of Social Eavesdropping in Communication Networks. International Journal of communication, 14. Obtenido de https://www.researchgate.net/publication/342572010_A_Model_of_Social_Eavesdropping_in_Communication_Ne tworks

Bouchard, L. (2017). Improving Board Knowledge with Information and Communication Technologies. Electronic Journal of Knowledge Management, 15(4), 215-224.

Cano-Pita, G. (2018). Las TICs en las empresas: evolución de la tecnología y cambio. Dominio de las Ciencias, 4(1), 499510. doi:http://dx.doi.org/10.23857/dom.cien.pocaip.2017.4.núm.1.enero.499-510

Castillo, G. A., Berbey, A., Torre, I., \& López, M. (2017). Information and Communications Technologies Health Projects in Panama: A Systematic Review and their Relation with Public Policies. Journal of medicales sistemas, 41(7), 101 - 125. doi:https://doi.org/10.1007/s10916-017-0755-y

Chaín, C., Muñoz, A., y Más, A. (2008). La gestión de información en las sedes web de los ayuntamientos españoles. Revista española de documentación científica, 31, 612-638.

Charry, H. (2018). La gestión de la comunicación interna y el clima organizacional en el sector público. Comuniacción, 9(1), 25-34. Recuperado el 28 de diciembre de 2019, de http://www.scielo.org.pe/scielo.php?script=sci_arttext\&pid=S2219-71682018000100003

Corona, J. (2016). Apuntes sobre método de investigación. Medisur, 14(1), 87-88. Obtenido de https://www.redalyc.org/pdf/1800/180044014017.pdf

Correa, M., y Diaz, B. (2018). Capacidad en tecnologías de la información y desempeño organizacional: un estudio en el contexto colombiano. Innovar, 28(69), 99-116. doi:https://doi.org/10.15446/innovar.v28n69.71699

Courtney, M., \& Steele, K. (s.f.). Mentorship in Collections and Resource Management: An Implied Competency. The Serials Librarían, 78, 93-97. doi:https://doi.org/10.1080/0361526X.2020.1731884

Culebro - Trujillo, R., Contreras, N., Montiel, A., \& Barragán, R. (2016). Satisfacción de los usuarios sobre las fuentes de información electrónica en un hospital de tercer nivel de atención médica. Educacion medica, 18(3), 188---194. doi:http://dx.doi.org/10.1016/j.edumed.2016.06.017

Díaz, E., Gorgoso, A., Sánchez, Y., \& Riverón, G. (2019). La dimensión didáctica de las tecnologías de la información y las comunicaciones. RITI, 8(15). doi:https://doi.org/10.36825/RITI.08.15.002 
El País. (2019). Acceso a la tecnología: la nueva desigualdad desde niños. Obtenido de Ediciones El País S.L.: https://elpais.com/elpais/2017/12/05/planeta_futuro/1512475978_439857.html

Fereshteh, F., \& Abbas, F. (2016). Accreditation of Management Communication and Information Systems in Public Hospitals of Sabzevar City, Iran. Informatica medica, 24(2), 124-129. Obtenido de https://www.ncbi.nlm.nih.gov/pmc/articles/PMC4851504/

Ferreira, M. M., De Lima, L., Vandresen, L., Gomes, M. J., Gomes, C. M., \& Lumini, M. J. (2020). Tecnologías utilizadas por los directores de enfermería en los hospitales portugueses. Revista Gaúcha de Enfermagem, 41(1), 1 - 10. doi: https://doi.org/10.1590/1983-1447.2020.20190294

García, A., Guzmán, T., \& Pons, L. (2019). Integración de tecnologías de información y comunicación en la enseñanzaaprendizaje de la enfermería. Tecnologia educativa, 4(2). Obtenido de http://tecedu.uho.edu.cu/

González, K. P., Tovilla, C. A., Juárez, I. E., y López, M. L. (2017). Uso de tecnologías de la información en el rendimiento académico basados en una población mexicana de estudiantes de Medicina. Revista Educación Médica Superior, 31(2), 1 - 10. Obtenido de http://scielo.sld.cu/scielo.php?script=sci_arttext\&pid=S0864-21412017000200008

Hanum, Y., Gogilavani, S., \& Mohamed, I. (2020). Predicting healthcare professionals' intention to use poison information system in a Malaysian public hospital. Health Inf Sci Syst, 3(8). doi:https://doi.org/10.1007/s13755-019-0094-0

He, D. (2019). Design and Implementation of Resource Information. Journal of Physics: Conference Series, 1345(5), 1-5. doi:10.1088/1742-6596/1345/5/052028

Herbas, B., y Rocha, E. (2018). Metodología científica para la realización de investigaciones de mercado e investigaciones sociales cuantitativas. Perspectivas (42). http://www.scielo.org.bo/scielo.php?script=sci_arttext\&pid=S1994$37332018000200006 \& \operatorname{lng}=\mathrm{es} \& \mathrm{nrm}=\mathrm{iso}$

Hernández-Leal, E.; Duque-Méndezy, N. y Moreno-Cadavid, J. (2017) Big Data: una exploración de investigaciones, tecnologías y casos de aplicación. Revista TecnoLógicas, 20 (39), 1-24. https://doi.org/10.22430/22565337.685

Hernández, F., Ramírez, R., \& Laguado, R. (2019). Communications management in the success of projects. Case study: Provincial university. Journal of Physics: Conference Series, 1388, 1-7. doi:10.1088/1742-6596/1388/1/012048

Hernández, R.; Fernández, C. y Baptista, M. (2014). Metodología de la investigación (6ºd.). México: Mc GrawHill. / Interamericana Editores, S.A. de C.V.

Iñiguez-Jiménez, S., Cruz-Pierard, S., y Vaca-Córdova, S. (2020). Uso problemático de tecnologías de la información y comunicación, consumo de sustancias y su impacto en la salud mental de estudiantes de bachillerato. Revista Científica Paideia XXI, 10 (2), 472 - 489. doi: https://doi.org/10.31381/paideia\%20xxi.v10i2.3448

Izhar, U., Ma, X., \& Aasir, I. (2017). Role of information \& communication technology (ICT) and e-governance in health sector of Pakistan: A case study of Peshawar. Cogent Social Sciences, 3(1). Obtenido de https://www.tandfonline.com/doi/full/10.1080/23311886.2017.1308051?src=recsys

Izzatty, Nurul, Hazana, N., \& Shamsuddi, A. (2015). Adoption of Hospital Information System (HIS) in Malaysian Public Hospitals. Revista Sience, 172, 336 - 343. Obtenido de

https://webcache.googleusercontent.com/search?q=cache:AokbcBiKWDcJ:https://core.ac.uk/download/pdf/82705 709.pdf $+\& \mathrm{~cd}=4 \& \mathrm{hl}=\mathrm{es}-419 \& \mathrm{ct}=\mathrm{clnk} \& \mathrm{gl}=\mathrm{pe}$

Jones, C., Motta, J., Alderete, M. (2016). Gestión estratégica de tecnologías de información y comunicación y adopción del comercio electrónico en Mipymes de Córdoba, Argentina. Estudios Gerenciales, 32(138), 4-13. doi:https://doi.org/10.1016/j.estger.2015.12.003

Lawan, A. (2019). The Impact of Information and Communication Technology (ICT) on Structural Adjustment in Public Service of Nigeria. Pakistan, Australia: Readers Insight Publisher. doi:edsbas.A8FFFEE8

León, L. (2018). Evaluación de las necesidades de implementación de tecnologías de información y comunicación en municipalidades no consideradas como ciudades principales: caso Oropesa-Cusco. Revista Científico Cultural Yachay, 7(1), 362-366. doi:https://doi.org/10.36881/yachay.v7i01.85

Martin, G., Arora, S. (2019). The impact of mobile technology on teamwork and communication in hospitals: a systematic review. Journal of the American Medical Informatics Association., 26(4), 339-355. https://doi.org/10.1093/jamia/ocy175

Martín, J. (2019). Gestión de la Comunicación: Un enfoque integral ( $1^{\mathrm{a}}$ ed.). Madrid: ESIC.

Martínez, M. (2018). Acceso y uso de tecnologías de la información y comunicación en México: factores determinantes. Revista de Tecnología y Sociedad, 8(14), 1 - 18. doi:https://doi.org/10.32870/pk.a8n14.316

Martin-Fumadó, C., Morlans, M., Torralbad, F. (2019). La comunicación del error médico. Consecuencias éticas y médicolegales. Medicina Clínica, 152(5), 195-199. doi:https://doi.org/10.1016/j.medcli.2018.07.013 
Medina, J. (2019). Gerencia Tecnológica de la Información y Comunicación en las Organizaciones Sociales. Revista Scientific, 4(12), 275-289. doi:https://doi.org/10.29394/Scientific.issn.2542-2987.2019.4.12.14.275-289

Mendoza, H., Placencia, M. (2017). Uso docente de las tecnologías de la información y comunicación como material didáctico en Medicina Humana. Investigación en educación médica, 7(26), 54 - 62. doi:http://dx.doi.org/10.1016/j.riem.2017.04.005

Ministerio de Tecnologías de la Información y las Comunicaciones de Colombia. (12 de noviembre de 2018). Fortalecimiento de la gestion en el estado: Gestión de información. Obtenido de MINTIC: https://www.mintic.gov.co/gestionti/615/w3-article-4796.html?_noredirect=1

Moreno, G., Herrera, J. L. (2019). Desarrollo de una arquitectura empresarial para mejorar la gestión de tecnologías de información en la Dirección General de Infraestructura, Equipamiento y Mantenimiento de Salud (DGIEM). Revista New Science, 1(1), 63-84. Obtenido de http://revistas.autonoma.edu.pe/index.php/NS/article/view/122

Muñoz, L., Díaz, E., Gallego, S. (2020). Las responsabilidades derivadas del uso de las tecnologías de la información y comunicación en el ejercicio de las profesiones sanitarias. Anales de Pediatría, 92(5), 307.e1-307.e6. doi:https://doi.org/10.1016/j.anpedi.2020.03.003

Muriel, J., García, M., López, N., \& Sánchez, M. (2019). Las tecnologías de la información y la comunicación. Revista de la sociedad española del dolor, 26(3), 175-183. doi:10.20986/resed.2019.3709/2018

Oliver-Mora, M., \& Rueda, L. (2017). El uso de las tecnologías de la información y la comunicación (TIC) en los centros de salud: la visión de los profesionales en Cataluña, España. Interface, 21(63), 945-955.

Papic, K. (2019). Los tipos de comunicación organizacional interna en establecimientos educacionales municipalizados en Chile. Cuadernos de Investigación Educativa, 10(1), 63-83. doi:http://dx.doi.org/10.18861/cied.2019.10.1.2881

Parra-Rodriguez, L., Pavón-Sicilia, Y., y Guerrero-Rodriguez, Y. (2016). Procedimiento de un sistema de comunicación para la gestión de calidad. Ciencias Holguin, 22(3), 1-17. Obtenido de https://www.redalyc.org/pdf/1815/181546432007.pdf

Pissaia, L. F., Kunz, A. E., Moreschi, C., \& Rempel, C. (2017). Tecnologias da informação e comunicação na assistência de enfermagem hospitalar. Revista de Epidemiologia e Controle de Infecção, 7(4), 1 - 5. doi: http://dx.doi.org/10.17058/reci.v7i4.8959

Plazzota, F., Luna, D., y Gonzáles, F. (2015). Sistemas de Información en Salud: Integrando datos clínicos en diferentes escenarios y usuarios. Revista peruana médica, 32(2). Obtenido de http://www.scielo.org.pe/scielo.php?script=sci_arttext\&pid=S1726-46342015000200020

Preciado, A., Hincapie, C., \& Pabón, M. (2019). Los indicadores de medida en la comunicación organizacional. Revista de comunicación, 121- 131. Obtenido de https://webcache.googleusercontent.com/search?q=cache:8MtU54XrSRQJ:https://dialnet.unirioja.es/descarga/artic ulo/3412501.pdf $+\& \mathrm{~cd}=1 \& \mathrm{hl}=\mathrm{es}-419 \& \mathrm{ct}=\mathrm{clnk} \& \mathrm{gl}=\mathrm{pe}$

Quispe, A., Padilla, M., y Telot, A. (2017). Rutas para una gestión estratégica y articulada de la información y la comunicación en contextos organizacionales. Revista de ingenieria, 38(1). Obtenido de http://scielo.sld.cu/scielo.php?script=sci_arttext\&pid=S1815-59362017000100008

Rivera, J., Herrera, V., Naranjo, X., \& Narváez, C. (2019). Gestión de Riesgos de TIC en hospitales públicos. RISTI, 20(5), 280-291.

Robledo, P. (2019). Impacto de la aplicación de las Tecnologías de la Información (TICs) en un modelo de atencion medica. Rev Sanid Milit Mex, 73(2), 96-104.

Rodríguez, Y., y Del Pino, T. (2017). Rutas para una gestión estratégica y articulada de la información y la comunicación en contextos organizacionales. Revista Cubana de información y comunicación, 6(14), 3-31. Obtenido de http://scielo.sld.cu/pdf/ralc/v6n14/ralc02317.pdf

Rubio, Z., Burrola, V., \& Corllaes, M. (2018). Modelo de gestión del conocimiento apoyado en las tecnologías de información y comunicación. Criterio libre, 16(28), 221-236.

Salas, B., Ocaña-Fernández, Y., Nieto-Gamboa, J., Alarcón, M. A., Luy-Montejo, C., \& Núñez, L. A. (2020). Communicative Skills as a Result of Successful Intelligence. Psychology and Education, 57(1), 62-69. Obtenido de http://repositorio.ucv.edu.pe/bitstream/handle/20.500.12692/43954/AC_Salas_VBJ-Oca\%C3\%B1a_FY-Nieto_GJAlarc\%C3\%B3n_DMA-Luy_MCA-N\%C3\%BA\%C3\%B1ez_LLA.pdf?sequence=1\&isAllowed=y

Sánchez, G., Montenegro, A., y Medina, P. (2019). Teletrabajo una propuesta de innovación en productividad empresarial. Digital Publisher, 4(5-1), 91-107. doi:https://doi.org/10.33386/593dp.2019.5-1.133

Sheykhotayefeh, M., Safdari, R., Ghazisaeedi, M., \& Mohammadzadeh, N. (2017). Hospital Information Systems 
Implementation: An Evaluation of Critical Success Factors in Northeast of Iran. Global Journal of Health Science, 9(2), 93 - 101. doi:https://doi.org/10.5539/gjhs.v9n2p93

Sotillos, B., Buiza, B., Herrera, Manuel, y Santana, V. (2018). Visión ciudadana sobre la prescripción de aplicaciones móviles de salud y el uso de tecnologías de la información y la comunicación en el entorno sanitario en Andalucía. Journal of Healthcare Quality Research, 33(4), 225 - 233. doi:https://doi.org/10.1016/j.jhqr.2018.04.004

Stanford, S., Schwartz, B., \& Khan, Y. (2020). The role of tacit knowledge in communication and decision-making during emerging public health incidents. International Journal of Disaster Risk Reduction, 50. doi:https://doi.org/10.1016/j.ijdrr.2020.101681

Suárez, A., Cuz, I. (2015). La gestión de la información: herramienta esencial para el desarrollo de habilidades en la comunidad estudiantil universitaria. Revista universidad y sociedad, 7(3), 72-79. Obtenido de http://scielo.sld.cu/pdf/rus/v7n2/rus10215.pdf

Torres, J., Gallo, J., Hallo, R., Jaraiseh, J., Muriel, M. \& Fernández, A. (2017). Gestión de la información como herramienta para la toma de decisiones en salud: escenarios más probables. Revista Cubana de Investigaciones Biomédicas, 36(3), 1 - 10. Obtenido de http://scielo.sld.cu/pdf/ibi/v36n3/ibi10317.pdf

Wagner, M., Bezaidenhout, L., Roos, P. (2014). Communication satisfaction of professional nurses working in public hospitals. Journal of Nursing Management, 23(8). doi: https://doi.org/10.1111/jonm.12243

Yánez, A., Ortiz, L., Espinosa, V. (2017). Las tecnologías de la comunicación e información (TIC) en salud: un modelo para aplicar en la carrera de Enfermería. Revista Iberoamericana De Educación E Investigación En Enfermería, 6(2), 29-36. Obtenido de https://www.enfermeria21.com/revistas/aladefe/articulo/200/las-tecnologias-de-lacomunicacion-e-informacion-tic-en-salud-un-modelo-para-aplicar-en-la-carrera-de-enfermeria/

Yang, Y., Saladrigas, H., Torres, D. (2016). El proceso de la comunicación en la gestión del conocimiento. Un análisis teórico de su comportamiento a partir de dos modelos típicos. Universidad y sociedad, 8(2). Obtenido de http://scielo.sld.cu/scielo.php?script=sci_arttext\&pid=S2218-36202016000200022

Yu, M. (2020). Standardization Construction of Fire Information Communication Operation and Management Based on Remote Communication. Journal of Physics: Conference Series, 1570(3), 1-6. doi:10.1088/1742$6596 / 1570 / 1 / 012048$ 